\title{
Texts as Data I : Document Analysis
}

\section{Karppinen, Kari}

Palgrave Macmillan

2019

Karppinen , K \& Moe , H 2019 , Texts as Data I : Document Analysis . in H Van den Bulck, M Puppis, K Donders \& L Van Audenhove (eds), The Palgrave Handbook of Methods for Media Policy Research. Palgrave Macmillan .

http://hdl.handle.net/10138/308912

submittedVersion

Downloaded from Helda, University of Helsinki institutional repository.

This is an electronic reprint of the original article.

This reprint may differ from the original in pagination and typographic detail.

Please cite the original version. 
This is a preprint of a chapter to be published in Van den Bulck, H., Puppis, M., Donders, K. \& Van Audenhove, L. (Eds.), The Palgrave Handbook of Methods for Media Policy Research. https://www.palgrave.com/gp/book/9783030160647

\title{
Focusing On Texts I: Collecting Policy and Industry Documents
}

Kari Karppinen, University of Helsinki

Hallvard Moe, University of Bergen

\begin{abstract}
Collecting and analyzing policy and industry documents is a basic part of most media policy research. The convenience of using existing material produced by public or private institutions can make the use of such documents seem relatively straightforward. However, the selection of relevant documents, their availability, collection and analysis also present methodological problems that researchers need to be aware of. In this chapter, we discuss different understandings of what "documents" are and review basic approaches to collecting and using policy and industry documents in media policy research. We then reflect on the advantages and hazards associated with collecting and using documents as research material. We emphasize the blurred nature of the distinction between "primary" documents and "secondary" interpretation, and point out that documents are always socially produced. All policy and industry documents frame issues in a certain light and only present one perspective into the issues and possible solutions, thus necessitating source criticism. Furthermore, the relative ease of accessing documentary sources does not mean that all potentially relevant documents (e.g. internal company documents) are equally readily available to researchers. In many contexts, access to documents can be deliberately blocked or limited. Using documents also entails limitations that stem from challenges with transparency and comparability. Finally, we note that documents leave silences: focusing on the visible exercise of official power, policy documents usually do not cover radical alternatives and policy options never considered in the first place. After discussing these challenges, the chapter presents a step-by-step process of collecting documents, from research design to accessing the documents, illustrated with the help of two cases studies.
\end{abstract}




\section{Focusing on Texts I: Collecting Policy and Industry Documents}

Policy and industry documents present an obvious starting point for media policy analysis. In one way or another - as the sole source of data, in combination with others, or as mere background material - those wanting to research media policy at some point face issues related to collecting policy and industry documents.

Online, policy researchers can search and access a range of publicly available documents with relative ease. The convenience of using existing material produced by public or industry bodies can make the use of documents seem self-evident and methodologically unproblematic (see Bowen, 2009; Karppinen \& Moe, 2012; Scott, 1990). Compared to other sources, such as interview or survey data, documents are right there and accessing them often costs nothing. However, the availability and ease of access to diverse documentary sources can create methodological challenges. Different research questions imply different criteria of relevant documents, not all relevant documents are readily available, those that are can give a partial or even misleading picture of policymaking, and much of what goes on in the policy-making process does not even leave a paper trail to be analyzed. As with any other methods, then, media policy researchers need to be aware of limitations and biases when relying on policy and industry documents as research material.

In this chapter, we first discuss different understandings of what documents are, illustrate the different types of policy and industry documents, and review basic approaches to document collection and analysis. We then reflect on the hazards associated with collecting and using documents as research material. Finally, the step-by-step process of collecting policy and industry documents is illustrated with the help of two cases studies. 


\section{Definition, logic and rationale}

How did the idea of regulating media ownership develop in the UK legal framework? Is "self-governance" understood differently across EU member states? To what extent is the regulation or taxation of internet companies such as Facebook and Google an issue on the transnational level, and what different solutions have been proposed? And how does the argumentation of NGOs differ from that of commercial lobbyists on any of these issues?

Policy and industry documents can help us gain an overview of policy issues and debates, the history and context of political and regulatory developments, the emergence of a new concepts, policy-making options, as well as different stakeholders and their positions and views. As such they can be employed in almost all types of media policy research. Different research interests and questions, however, involve different demands on what types of documents may be considered relevant.

To begin with, there are different conceptions in social science concerning what a document is. In a much-quoted textbook on documentary sources, John Scott defines a document as "an artefact which has as its central feature an inscribed text" (1990, p. 5). Others, inclined to think of texts as media more broadly, also include audio-visual sources in their definition of documents (see Karppinen \& Moe, 2012). Documents are often explicitly distinguished from "research literature", as records produced or generated without a researcher's intervention (Bowen 2009, p. 27). Documents can also be categorized according to whether they are made systematically or accidentally, whether they were intended for a public readership, and whether they belong to the realm of research or not (Schade, 2007).

Broadly understood, documents include a diverse range of different types of sources, including not only written reports but also letters, diaries, pictures, videos, speeches, and so forth. In the context of public policy analysis, however, the policy and industry documents are often 
understood more narrowly to mean official documents, such as legal texts, background reports, position papers, meeting agendas, press releases, consultations and memos produced by either public sector agencies or other stakeholders, like companies, industry interest groups or other civil society organizations - in other words, "documents that affect a public decision making process" (Syvertsen, 2004, p. 216).

It is also useful to make a distinction between interest in documents as sources and as objects of study. In the first case, documents are understood as factual or contextual sources (akin to historical research) that can reveal the interests and intentions of their authors or in other ways uncover facts about the policy process. In the second case, documents are treated as meaningful social products or cultural artefacts that have independent consequences and are worth analyzing in themselves. In either case, using documents implies some form of textual analysis (Karppinen and Moe, 2012). While this chapter focuses on collecting documents, issues related to their analysis are treated in later chapters of this handbook (see chapters $\mathrm{xx}$ ).

In this chapter, we focus particularly on the most typical policy and industry documents, as other types of documents, such as media texts, websites, statistics, and television archives are discussed elsewhere in the handbook. The porosity of borders is important, though, especially as policy actors make use of new forms of communication (e.g. social media memes) or reinterpret old ones (e.g. politicians' autobiographies). Even in our narrower context, however, it is clear that the types of documents that can be relevant for media policy research cover a broad range, not all of which can be exemplified here.

\section{Critical assessment of the method}

There are a number of important advantages with using documents. Compared to most other sources, documents are stable: they can be stored, retrieved and copied. Many documents 
are even easily available, and they give researchers first-hand access to policy processes and stakeholders' positions. If the alternative is either asking people involved or observing the actual processes, documents seem more realistic to use, and sometimes, for instance when dealing with historical issues, the only means. Documents are therefore an efficient and cost-effective data source (Bowen, 2009).

There are also a number of important challenges with using documents: Documents are often considered distinct from academic literature and other commentary or secondary sources that offer "interpretation". As "primary sources" that represent objective statements of facts, written by active participants in the policy process, documents are understood as more reliable sources of factual information about policy processes (see Karppinen \& Moe, 2012). In practice, however, all policy and industry documents - no matter how dry and neutral - frame issues in a certain light, and only present one possible construction of reality and one perspective into the issue and possible solutions. Although official documents are often read as objective statements of facts, they are always socially produced.

At a fundamental level, documents raise the issue of authenticity. Documents can be falsified or tampered with; they can include mistakes, omissions and distortion; and they might be more or less typical of their kind. As policy researchers like Kingdon (2003) have emphasized, policy-making is not a unidimensional, coherent process, but messy and chaotic. A large number of actors and ideas float around in the "primeval soup" of policy alternatives, and solutions that are chosen are contingent on many factors. Reconstructing the policy process retrospectively on the basis of policy and industry documents thus involves the danger of overestimating the rationality and linearity of the policy-making process.

They way to address these issues is by source criticism. A document is a social phenomenon in the sense that it was created under a set of circumstances, for a specific purpose, 
by specific people, at a certain time and place (Syvertsen, 1992, p. 54). We need to question both the authenticity (indisputable origin) and the credibility (error and inaccuracies) of each source. We also need to consider how representative a source is or, alternatively, clarifying how it is atypical (Scott, 1990, pp. 6-8). Furthermore, researchers also need to document the process of selecting the data, maintain a critical relation to the sources, and be transparent regarding their normative premises and own possible stake in the policy process. Source criticism and selfreflection is not an isolated step in the research process, but a constant, guiding also in our interpretations of the documents (see also Reh, 1995).

While there are more and more documents available online, a lot of potentially relevant documents are not readily available to researchers, and sometimes access to documents can be deliberately blocked or limited. Draft versions of policy documents shared between government agencies or strategic documents of media companies, for example, would likely interest researchers but are often not available. There are major differences in transparency and access to documents between policy-making contexts and across public and private organizations. It may sometimes be necessary to actively request access to particular documents or information under freedom of information laws. In such cases, the ease of access (in terms of extent, speed and costs) greatly varies between countries with different laws and political cultures (e.g. Banisar 2006; Erkkilä, 2012). Sometimes, researchers may also rely on more exceptional sources, such as leaked documents, which can trigger additional ethical considerations.

Further challenges lie with documents' varied nature. Documents can also be hard to contextualize and interpret, or more fundamentally, they can be written to address different audiences and to serve different purposes with consequences for their argumentative style, the use of references and substantiation of claims. This creates challenges when piecing together a comprehensive picture of the policy process, and in particular when making comparisons. How 
should, for instance, major publishers' press releases or annual reports be analyzed and contextualized in relation to a government act? And how do you compare such documents from France and Brazil?

The way to address this challenge is to understand the limitations in making comparisons (see chapter $\mathrm{xx}$ ) and to familiarize oneself with the political and cultural contexts, in which the documents have been produced. Often it may also be necessary to corroborate and complement official documents by means of other methods (triangulation or mixed methods design).

A final pitfall associated with documents is that they do not cover alternatives and policy options never considered in the first place. As Des Freedman (2010) has noted, media policy research usually focuses on the public exercise of official power, visible and overt decisionmaking actions like government intervention, regulatory activity, civil society engagement, and corporate initiatives. Relying on official documents - or letting these documents guide the premises and parameters of the research - thus involves the danger of neglecting more radical alternatives or less visible arenas and modes of decision-making and power.

According to Freedman, to take into account the processes of "policy silences" and "nondecision making", policy researchers need to examine also "the means by which alternative options are marginalized, conflicting values delegitimized, and rival interests derecognized" (Freedman, 2010, p. 347). This is another reason why it is often useful to complement and compare official documents with other sources, such as rival accounts produced by civil society organizations and activists, academic debates, media coverage, or other sources that clarify the broader historical and political context.

The process of identifying and accessing the most relevant documents depends entirely on the aims of the study: In a study that compares legislation in different countries, it may be sufficient to simply collect the relevant legal texts (and possible translations). A study that 
attempts to uncover the policy formulation process and the power of different influences, on the other hand, needs to seek access to public consultations and position papers, and perhaps also lobbying records or other correspondence between stakeholders and policy-makers. Finally, for a study that focuses on the framing and discourses around policy issues, it may be reasonable to concentrate on other type of texts, such as speeches, media coverage, parliamentary debates or press releases. In addition, it is often useful to complement documents with interviews with policymakers or stakeholders, surveys, participant observation, or other methods depending on the research questions.

\section{Planning and conducting: a hands-on 'how to' guide}

In the following, we illustrate the methodological steps with the help of two case studies. We distinguish three main phases involved in using policy and industry documents in media policy research: (1) the research design and identification of potentially relevant document types and sources; (2) accessing, collecting and sampling of the documents; and (3) conducting the analysis (for a more detailed description of the research process, cf. Altheide \& Schneider, 2013).

The last step will be discussed in more detail later in the handbook, but we will here briefly touch upon how different methods of analyzing documents also have implications for the choice of relevant documents.

Both cases touch upon the role of public service media, and how policies change as old institutions face new media. But the cases differ in key aspects for our illustrative purposes: one is comparative (Finland vs Germany), the other is a single-country case study (Norway), and they illustrate how different research questions and theoretical assumptions lead to different emphases and priorities regarding document selection. 


\section{Case study 1}

In 2013, Germany and Finland made the switch from the traditional public broadcasting licence fee tied to television-set ownership to a compulsory excise duty collected from all citizens, households and places of business, irrespective of any device. In both cases the funding reform constituted a major media policy issue with significant implications for the relationship between the state, the market, the public service media system and licence-fee contributors. In their study of the policy processes that preceded the decisions, Herzog and Karppinen (2014) compared the reforms on the basis of Kingdon's "multiple streams" framework of public policymaking. Drawing on the analysis of policy documents and interviews with policymakers and other stakeholders involved in the respective processes, the study investigated how the actual reforms materialized, which other possibilities were neglected and why this was the case. In particular, the study paid attention to the influence of policy-making traditions and the role of industry and other stakeholders in opening a "window of opportunity" for major reforms.

\section{Case study 2}

Public service broadcasting has always been criticized for failing to be of service to the public. The criticism operates on different levels. On one level, some claim that broadcasting, including the public service version, has never addressed the public but, rather, an audience of individual, private consumers. On another level, critics follow activists fighting for greater participation - ultimately for radio and television as a two-way medium. Only then, the argument goes, will the public truly be served. With the advent of the digital era, public service institutions made use of new opportunities to tackle such criticism, e.g. by initiating extensive internet 
activities made up of user-generated content. The success or failure of such initiatives depend neither on institutional strategies alone nor the extent to which specific organizations are able to translate ideas into actual applications. Public service broadcasting is a tool for media policy. If the prominence ascribed to the users is to have lasting consequences, the ideas need to rub off on actual policy. Based on that presumption, Moe (2010) used Norway as a case and asked what notions of "the public" could be found in public service broadcasting policy for the digital era. Building on deliberative democratic theory's ideas of the relations between the public and the media, the analysis was especially focused on to what extent the public was considered as a factor for policy change, and whether the users were envisioned as participants.

\section{Step 1: Research design and the identification of relevant documents}

As noted above, the selection of which types of documentary sources are relevant for a given research project depends on the objectives. Formulating the aims of the study therefore precedes the actual collection of documents. The overall research design informs the strategy of choosing, collecting and analyzing the material and ensures that the different components of the study (research problem, theoretical framework, method) fit together in a coherent way. It is also important to consider whether the overall aims of the project are mainly descriptive, interpretative, causal, or critical/normative.

In practice, the research process is often recursive, in a sense that the availability of relevant documents or the emergence of new information or themes in the material may force us to revisit the initial research design and its focus. Therefore, the first stage of formulating a specific strategy can already involve extensive consultation of background material, including previous academic research, media and political commentary, and policy documents. One of the 
basic functions of policy documents in a research process is to suggest new questions that need to be researched (Bowen 2009, p. 30).

In both of the case examples discussed here, for example, the researchers had already followed (and occasionally participated in) the relevant policy and academic debates for years. The actual formulation of research questions was thus preceded by extensive reading of academic and political debates. This is often the case, so in practice the collection of documents does not start from scratch. This also illustrates how the distinction between primary and secondary documents may not always be clear-cut. Yet even if the process is not linear, it is important to explicate the initial assumptions of the study that guide document selection in order to allow readers to assess the validity of the findings and arguments.

In our first case, the study by Herzog and Karppinen (2014), the researchers were mainly interested in comparing dynamics of the policy processes and the influence of various stakeholders, policy-making traditions and political events in determining policy outcomes. Guided by a particular theoretical framework of "policy streams", and the aim to compare similarities and differences between two countries, the researchers focused their attention on documents that reveal the course of political events, the role of different stakeholders, and the emergence of different policy options within the policy-making community. The relevant documents in this case consisted of government documents: in particular, the reports of the working groups commissioned to prepare the public service funding reforms and present recommendations, the hearings and consultation processes associated with these, as well as other political and legal comments by various stakeholders. Documents were mainly collected from 2009-2012 to cover the active stages of the policy process, although historical context and previous debate in both countries were also taken into account to contextualize the process. A range of other sources, including media coverage and academic commentaries we used to 
establish the context and access different interpretations of the course of events. In addition, semi-structured interviews with policymakers, broadcasting officials and industry representatives were carried out to complement document analysis.

Our second case, the study by Moe (2010), by comparison, was more focused on meaning-making, and different interpretation of one central concept. This interest was guided by, on the one hand, an observed development in the media towards marketization, coupled with a new focus on the media audience as participants in the digital era. On the other hand, the interest came from theoretical conceptualizations of the role of the media for democracy, in which the media users as a public were seen as central. The document sources were chosen to empirically study the gaps between these domains.

This brings out differences in the role of documents in the two cases. In the first case study, it was paramount to piece together a chronological understanding of the policy process from initial ideas and discussions to formal decisions. Hence, the researchers used varied documents, complemented with interviews, to draw out interests and intentions from different stakeholders. In the second case, the documents served as the only empirical data, and they were analyzed not primarily to reveal a policy process, but to discuss the ways in which a key concept (the public) was framed and understood in media policy. Whereas the first case relied on documents more as factual sources, the second case approached documents more as texts worthy of analysis in themselves. In other cases, it is of course also possible to combine these approaches.

The approach to documents in each case was guided by the aims of the research project and their respective theoretical starting point. In any study, explicating the research problem and the initial assumptions of the study that lead to a particular approach is paramount to allow readers to assess these choices and their limitations. 


\section{Step 2: Access, collection, and sampling}

Because it relies on already existing sources, document analysis often requires more data selection than data collection (Bowen, 2009). Researchers will inevitably need to make decisions on which organizations and time frames to include. Both our cases relied on publicly available documents, so access as such was not a major challenge. In the study by Herzog and Karppinen, there was no shortage of potential research material with the reforms in question being subject to extensive media coverage, public debate, and policy deliberation. To delimit the material and to allow comparison, the analysis focused on key political events and identified central documents, which in both countries included government commissioned reports, their background material and comments to them. This involved a decision to exclude a range of other processes as well as previous policy decisions on the same issue. Another consideration was the comparative context, which guided the researchers to seek similar types of documents around chronologically close events. A particular challenge with this was that although the reforms followed a seemingly similar path in Finland and Germany, differences in the political systems (e.g. Germany having a federal system) and political cultures (e.g. the role of expert recommendations or parliamentary working groups) had to be taken into account in selecting the documents. The need to gain further insights into the policy process that do not show up in the documents was also one of the reasons why the study was complemented with interviews. Interviews thus aided in source criticism, as a means of establishing the context and origin of the documents collected. Besides authenticity and credibility, key issues for source criticism in this case involved making judgements on the status of given documents, their role in the policy processes, their intended audiences, and the aspects that publicly available documents may have omitted.

A typical sampling strategy for choosing the documents to analyze involves limiting the analysis to key events or a particular time frame. The latter strategy was chosen in our second 
case study: The possibility of policy documents to afford a historical perspective led to a fairly long period - from the advent of the digital era to the time of study. In order to collect comparable documents, i.e. documents that had a similar status and function, through this period, Government White Papers from one country were chosen. In Norwegian policy making, a White paper is written by a ministry (in this case the Ministry of Culture), and submitted to Parliament. It is often, but not always, proceeded by independent commission reports. White Papers are treated by the relevant committees in Parliament, and then debated before they (in full or part) end up as actual policy. For the case study, the most central White Papers on media policy in the period were chosen. The objective was to sort out more peripheral discussions of public service broadcasting and of the users as a public. Since both authenticity and credibility was fairly straight forward to establish in this case, source criticism focused on clarifying the characteristics of each individual text, especially the settings in which they were produced: rather than being chosen for their representativeness, each White Paper comes with the biases, views and preferences of the Government in office at the time, and inscribed with the technological dilemmas high on the agenda at each point in time. Thus, understanding and describing the context of each document was key to use of the documents, as well as to the analytical process (cf. Reh 1995).

Again, challenges related to accessing and sampling documentary sources vary depending on different research problems, political context, and different types of documents. This also depends on the method of analysis: if documents are analyzed using a systematic method of textual analysis, for example, it becomes essential to define the corpus beforehand, but if a researcher is using documents more as a source of background information or for tracing historical facts, it is possible to adopt a more open-ended approach where new documents can be included as they are uncovered. In any case, key considerations for researchers are the possible 
biases and limitations that the material entails, and the need to document the process and rationale of data collection as transparently as possible.

\section{Step 3: Analysis and impact}

Thirdly, in terms of analysis, our two case studies also represent different approaches to documents as texts. As Herzog and Karppinen's aim was to analyze and re-interpret a chain of political events and their contextual factors, they were not particularly interested in the discursive analysis of the documents, but more in identifying different policy actors and tracing their activities and positions. On the other hand, in Moe's study, documents were more used as texts, to trace meanings and compare them with the understandings of the same key concept (the public as participants) found in scholarly works. One important limitation with this approach was the relatively few documents: by limiting the analysis to one kind of document (White papers), with substantial discussion of the topic in question, in a single country. Another challenge here lies in the specific function of White papers. A White paper does not necessarily follow a logically constructed chain of argumentation. Rather, it is a presentation of related issues deemed relevant, with mentions of views of these issues. They are seldom meant to be read from start to finish like

a book, but rather to be checked for reference. Such genre characteristics are important to keep in mind when designing a research project using documents.

In each case, it is important to note that other theoretical frameworks or different research aims could easily have led researchers to approach the same policy issues differently. It is always important to ask whether other researchers relying on other methods would have reached the same conclusions, and to what extent the results of the study can be generalized.

Both cases also illustrate the challenges with relying on publicly available documents as research material: An attempt by Herzog and Karppinen to trace the policy processes and 
influences, for example, remains only one interpretation of the events, and there is no certainty that the documents analyzed revealed all the underlying factors that contributed to the outcomes not to mention the options never considered in the first place. Causal relations are not readily reconstructed from policy and industry documents only. This applies to much of policy research, and one key lesson here thus concerns the need to be transparent about the limitations of any single study.

\section{Conclusion}

Policy and industry documents offer an efficient, cost-effective means to access both contemporary and historical media policy debates. However, with the range of possible analytical approaches and forms and types of documents available, it is important to consider which types of documents are most relevant for the specific research problem at hand. Researchers also need to be aware of the limitations and biases that different types of documents entail. The selection of documents and their relevance need to considered in relation to aims and theoretical approaches as well as practical considerations like availability. Often the most obvious or the most easily accessible documents are not the most fruitful.

It is also important to keep in mind that, despite the availability of a range of documentary sources, access to information, even basic government documents, remains limited and often blocked in many parts of the world. Even in countries with relatively open and transparent governance structures, it is always necessary to consider to what extent the documents available may provide a selective picture of the policy process and present only those facts that are favorable to their authors, and what kinds of omissions or silences they may involve.

None of these challenges, however, are reasons to avoid using documentary sources to address ambitious research questions involving cross-national comparisons, explanations of policy influences or normative judgments. Many of the problems discussed in the chapter can be 
addressed by following a systematic and transparent process of data collection, exercising critical self-reflection and source criticism, and by complementing documents with other methods.

Not all limitations can be overcome. Therefore, researchers will also need to be transparent about the limitations of their data and to document the process of data gathering, so other researchers can assess gaps and needs for further research. The use of policy and industry documents is often considered ethically relatively unproblematic in comparison to methods such as interviews or participant observation. Even when using publicly available documents, however, documentary research is not only neutral value-free observation but also involves normative choices and critical reflection. This requires also self-reflection and transparency on the part of the researcher and her motives and aims. 


\section{Referencesišrepi]}

Altheide, D. \& Schneider, C. (2013). Process of Qualitative Document Analysis'. In D. Altheide \& C. Schneider (Eds.), Qualitative Media Analysis, Second Edition. London: Sage.

Banisar, D. (2006). Freedom of Information around the World 2006. A Global Survey of Access to Government Information Laws. Privacy International. Available from: http://www.freedominfo.org/documents/global_survey2006.pdf

Bowen, G. A. (2009). Document Analysis as a Qualitative Research Method. Qualitative Research Journal, 9(2), 27-40. doi: 10.3316/QRJ0902027

Erkkilä, T. (2012). Government Transparency. Impacts and Unintended Consequences. Basingtoke: Palgrave Macmillan

Freedman, D. (2010). Media Policy Silences. The Hidden Face of Communications Decision Making. The International Journal of Press/Politics, 15(3), 344-361. doi:10.1177/1940161210368292

Herzog, C. \& Karppinen, K. (2014). Policy streams and public service media funding reforms in Germany and Finland. European Journal of Cmmunication, 29(4), 416-432.

Karppinen, K. \& Moe, H. (2012). What we talk about when we talk about document analysis. In N. Just \& M. Puppis (eds.), Trends in Communication Policy Research New Theories, Methods and Subjects. Bristol: Intellect.

Kingdon, J. (2003). Agendas, Alternatives, and Public Policies (2nd edn). New York: Longman. Moe, H. (2010). Notions of the Public in Public Service Broadcasting Policy for the Digital Era. In J. Gripsrud \& H. Moe (Eds.), The Digital Public Sphere: Challenges for Media Policy (pp. 99-116). Gothenburg: Nordicom. 
Schade, Edzard (2007). Audio- und Videodokumente als Quellen für die Kommunikations- und Medienforschung. In K. Deggeller et al (Eds.) Gehört-gesehen. Das audiovisuelle Erbe und die Wissenschaft (pp. 49-63). Baden/Lugano: Hier+Jetzt.

Reh, Werner (1995). Quellen- und Dokumentenanalyse in der Politikfeldforschung: Wer steuert die Verkehrspolitik? In U. von Alemann (ed.) Politikwissenschaftliche Methoden. Grundriss für Studium und Forschung (pp. 201-259), Opladen: Westdeutscher Verlag.

Scott, J. (1990). A Matter of Record. Documentary Sources in Social Research. Cambridge: Polity.

Syvertsen, T. (1992). Public Television in Transition (Vol. nr 5/92). Oslo: Norges allmennvitenskapelige forskningsråd.

Syvertsen, T. (2004). Mediemangfold - Styring av mediene i et globalisert marked. Kristiansand: IJ-forlaget. 